\title{
Photostimulable X-Ray Storage Phosphors: a Review of Present Understanding
}

\author{
Heinz von Seggern \\ Darmstadt University of Technology \\ Materials Science Division \\ Department for Electronic Materials \\ Petersenstraße 23 \\ D-64287 Darmstadt, Germany \\ Received 30 March, 1998
}

\begin{abstract}
Photostimulable $\mathrm{x}$-ray storage phosphors in form of image plates are a promising alternative to conventional two-dimensional x-ray detectors. By absorption of ionizing radiation, electrons and holes are generated and captured locally to form a dose proportional latent image. The stored information is read out by scanning with a focussed HeNe laser. This leads to a local excitation of the trapped electrons which subsequently recombine with the trapped holes causing the emission of light. The information is then recorded by means of a photomultiplier, digitized by an A/D converter and displayed with the aid of a computer. The present state of the field of storage phosphors is reviewed in the following areas: the image principle, today's understanding of the nature of the storage centers, their physical generation, and the optically stimulated charge transfer paths. In addition, improvements and applications of these phosphors will be discussed for existing and future commercial devices.
\end{abstract}

\section{Introduction}

Since its invention by C.W. Röntgen a little more than 100 years ago a vast number of different detection methods for $\mathrm{x}$-rays and generally ionizing radiation have been realized for application in medicine, physics, biology and materials science. In 1932 Professor Bernhard Gross wrote his first article on ionizing radiation [1] about the pressure dependence of ionization of cosmic rays which finally led to the well known Gross transformation [2]. The ionizing radiation and its impact on matter has since then accompanied Bernard Gross through his still ongoing scientific life. In 1941 B. Gross published an article on the theory of ionization chambers [3] followed in 1948 by an article on a new type of Geiger-Mueller counter [4], followed again in 1960 to 1961 by a series of articles on Compton dosimeters [5-9]. This work was also granted a US-patent with the title "Method and apparatus for measuring the dosage of $\mathrm{x}$ rays and gamma rays" [10]. Thereafter Gross became interested in the phenomenon of radiation induced conductivity in dielectrics which resulted in a series of papers from 1964 about gamma induced currents in Teflon [11] to 1989 about time-resolved x-ray induced conduc- tivity in PET[12]. Today Gross is interested and actively participates in work about secondary emission induced by low energy electron-beam irradiation which results in a very interesting contribution to the present issue of this journal [13].

The work presented below on storage phosphors as two dimensional radiation detectors contains the ideas and inspirations I experienced in my exciting time of collaboration with Bernhard Gross [14-18]. The radiation induced generation of charge carriers and their subsequent storage in radiation induced defects is a common language which connects the work of Prof. Gross with the storage phosphors for image plates.

In all detection methods either the energy distribution of the radiation or the lateral distribution of intensity, or both, are of major concern. The energy distribution of the radiation is mainly obtained by gas and solid-state ionization chambers [3]. In this case the number of generated charge carriers per absorbed $\mathrm{x}$ ray quantum is counted. One of the most severe problems with this technique is the overall limited counting rate which is caused by a temporal overlap of two or more ionization events or by the time-resolution of the electronic equipment. In the case of the determi- 
nation of the lateral intensity distribution, the objectpenetrating radiation is recorded either in an x-ray film, a film/screen system or a wire chamber.

The most widely known technique is the $\mathrm{x}$-ray film. In medicine the film/screen system is used solely due to its improved sensitivity compared to the x-ray film $[19,20,21]$. Due to its relatively small thickness the $\mathrm{x}$ ray film exhibits a high spatial resolution, but its sensitivity is low. Its dynamic range is restricted because the film structure contains sensitive Ag-halide grains of about one micrometer in size. Once such a particle has absorbed an x-ray quantum it will turn black during processing and no further absorption generates a measurable difference. Therefore the total dose such a film can record is restricted to the total number of $\mathrm{Ag}$ halide grains. The range of useful flux is 2 to 3 orders of magnitude in x-ray dose to be registered.

In the film/screen system, which is utilized mainly in medical diagnostics the low sensitivity of the $\mathrm{x}$-ray film is compensated by using a sandwich structure of a scintillating phosphor screen in combination with a photographic film. In this case the radiation is strongly absorbed by the phosphor screen (intensifying screen) and converted with up to $20 \%$ energy efficiency into visible light. This light is then absorbed by the photographic film which is the actual storage medium. In this way one gains the necessary sensitivity required for medical application, but one looses the high spatial resolution of the original $\mathrm{x}$-ray film due to light scattering processes in the phosphor film.

The image plate was introduced in 1983 as a new $\mathrm{x}$-ray detector system [22]. The image plate was built in analogy to the intensifying screen and consists of a polymeric support film covered by a layer of an organic binder in which the functional phosphor grains are imbedded. Contrary to the intensifying screens, the principle of these image plates is not the radiation induced generation of spontaneous luminescence but the radiation induced photostimulable luminescence (PSL). In this case the radiation generated electrons and holes are captured in electron and hole traps in the immediate surrounding of their generation. Proportional to the density of these trapped carriers the image plate contains a latent image of the $\mathrm{x}$-ray radiation dose. The information readout occurs by local optical excitation (photostimulation) in which the trapped electrons are liberated. These electrons then radiatively recombine with trapped holes under the emission of light.
The intensity of this emitted light is proportional to the density of trapped electron/hole pairs and thereby to the locally absorbed x-ray dose. Commercially applied storage media are the alkaline-earth halides $\operatorname{BaF}\left(\mathrm{Br}_{x}, \mathrm{I}_{1-x}\right): \mathrm{Eu}^{2+}$ with an added fraction (1-x) of iodine ions and the alkaline-halide RbBr:Tl${ }^{+}$. Further potential candidates with excellent storage and readout properties are $\mathrm{Ba}_{2} \mathrm{~B}_{5} \mathrm{O}_{9} \mathrm{Br}_{\mathrm{Eu}^{2+}}{ }^{2+} \mathrm{Ba}_{5} \mathrm{GeO}_{4} \mathrm{Br}_{6}: \mathrm{Eu}^{2+}$ [23], $\mathrm{Y}_{2} \mathrm{SiO}_{5}: \mathrm{Ce}^{3+}$, and $\mathrm{Y}_{2} \mathrm{SiO}_{5}: \mathrm{Ce}^{3+}, \mathrm{Sm}^{3+}$ [24].

After a short introduction to the principle of image generation and readout with $\mathrm{x}$-ray storage phosphors, the physical mechanisms of $\mathrm{x}$-ray storage phosphors will be discussed. Of interest are the nature of the electron and hole traps, the generation of these traps during irradiation, and the mechanism of information readout by means of photostimulated luminescence. Further, developments show ways to improve the parameters of the standard storage phosphors such as shorter readout time, a higher sensitivity and a better spatial resolution. At the end, an overview of existing and future applications of these interesting materials will be given.

\section{Imaging with image plates}

The process of imaging with image plates consists of three parts

1. the $\mathrm{x}$-ray exposure,

2. the information readout, and

3. the optical erasure of the residual information

During the exposure (Fig.1a) the ionizing radiation penetrating an object will expose the image plate. It generates a latent image consisting of trapped electronhole pairs in the $\mathrm{BaFBr}: \mathrm{Eu}^{2+}$ phosphor grains of the image plate. The readout is performed by means of photostimulated luminescence (PSL) by a HeNe-laser beam which scans the image plate point by point. The trapped electrons are thereby liberated and recombine with the trapped holes under the emission of $\mathrm{Eu}^{2+} \mathrm{lu}^{-}$ minescence. The emitted photons are collected in a photomultiplier which converts the luminescence into an electrical signal (see Fig. 1b). This signal, correlated with the scanning laser spot, is digitized by means of an $A / D$ converter and submitted to a computer for the reconstruction of the image. The remaining information in the image plate will then be erased by a strong halogen lamp (see Fig. 1c) so the image plate can be reused. 


\section{$\begin{array}{ll}\text { a) } x \text {-ray exposure } & \text { b) image readout }\end{array}$}

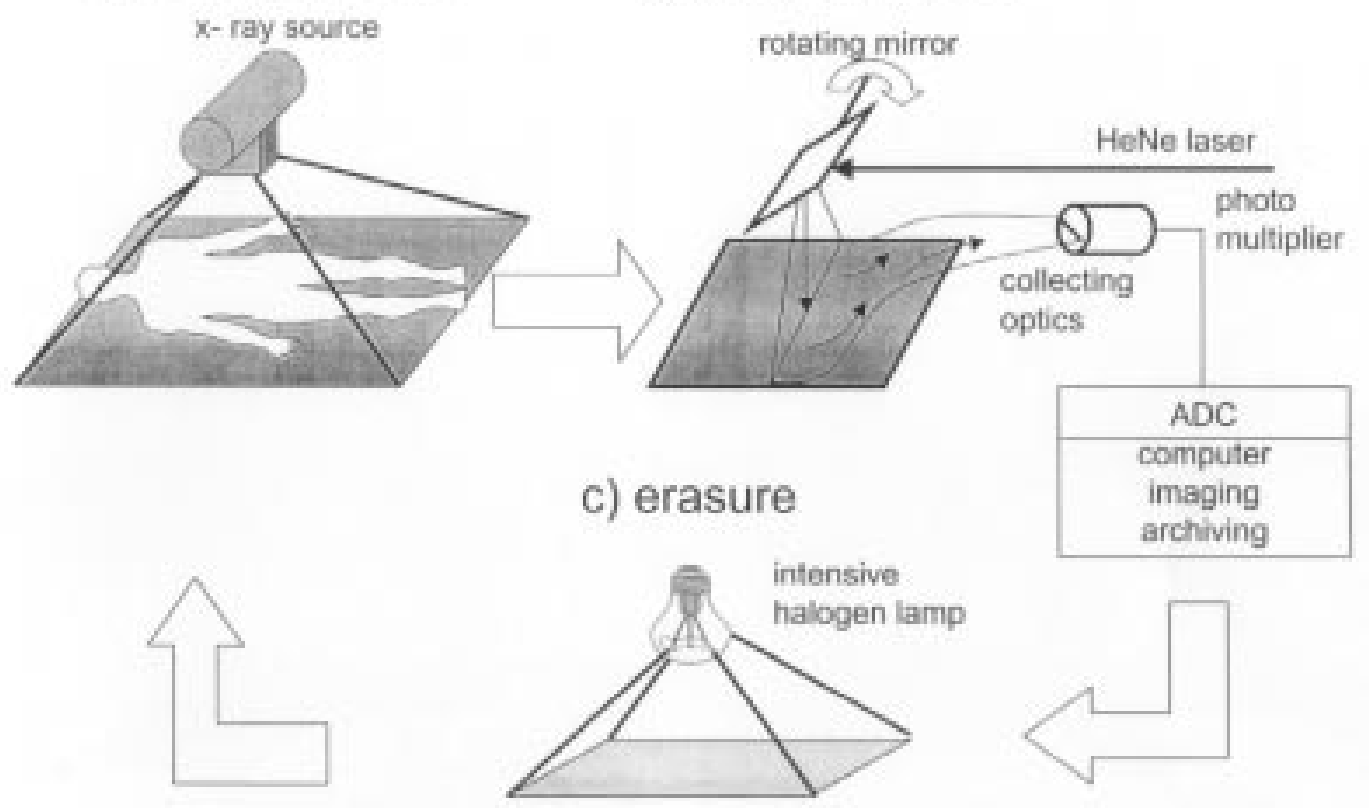

Figure 1. Schematics of the imaging process utilizing image plates.

Image plates have the following advantages over other techniques:

- dynamic range larger than 5 orders of magnitude in x-ray dose,

- lower limit of useful dose compared to the x-ray film,

- reusability,

- no wet chemical processing,

- images are available in digital form,

- image processing and pattern recognition possible, and

- simple data storage on optical or digital media

Disadvantages of image plates compared to the conventional $x$-ray film are a poorer spatial resolution due to light scattering at the storage phosphor grains during the readout process.

\section{Physical mechanism for BaFBr:Eu ${ }^{2+}$ storage phos- phors}

\section{III.1 Physical nature of electron and hole storage centers}

$\mathrm{X}$-ray storage materials contain at least two different storage centers: one for electrons which capture the $\mathrm{x}$-ray-generated electrons and release them by photostimulation, and hole storage centers, called activators, which capture the $\mathrm{x}$-ray-generated holes. In addition, these hole storage centers have to be able to capture an optically liberated electron and release the available energy to a luminescence center.

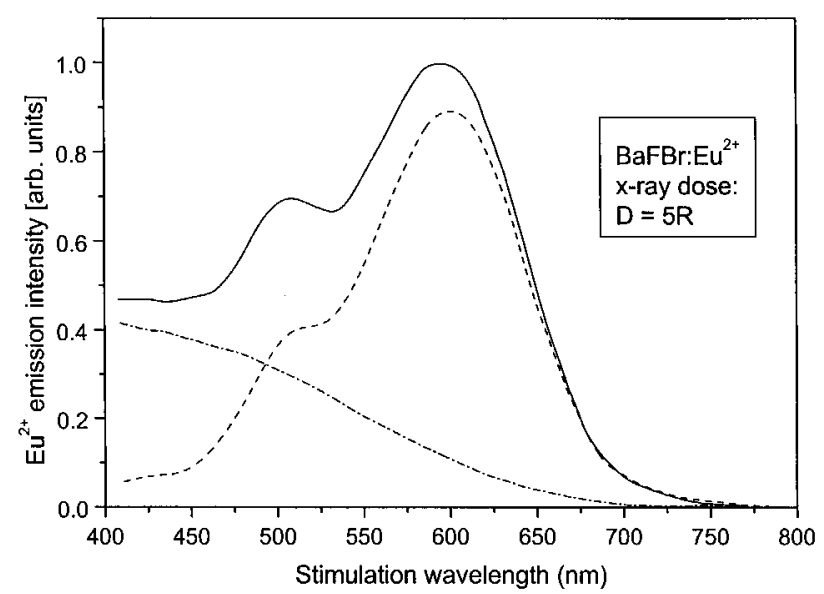

Figure 2. PSL spectrum of $\mathrm{Eu}^{2+}$ emission of a sample which was x-ray irradiated at room temperature (solid line); the dashed line resembles the stimulation spectrum of the $\mathrm{F}\left(\mathrm{Br}^{-}\right)$center obtained by the difference spectra after optical bleaching at $625 \mathrm{~nm}$. The dot-dashed line indicates a photostimulation center which has not been identified. 
It is known that in halide crystals, F-centers (Farbzentren) are generated by x-ray irradiation [25]. These centers are anion vacancies occupied by electrons. They are optically active displaying an oscillator strength close to one. In BaFBr crystals two types of $\mathrm{F}$ centers are known, one based on a fluorine-anion vacancy and the other based on a bromine-anion vacancy [26]. Both types of centers are generated, however, only the bromine based centers act as occupied storage centers contributing significantly to the photostimulation process [27]. For the identification of the F-center the so called photostimulation spectrum is used. This spectrum was obtained by tuning the excitation wavelength from 400 to $800 \mathrm{~nm}$ and detecting the intensity of the $\mathrm{Eu}^{2+}$ emission band at $390 \mathrm{~nm}$. The obtained spectrum resembles the spectral excitability of the PSL process and agrees in its shape to the absorption spectrum of the F-center. Such an agreement was verified explicitly for the storage phosphor $\left.\mathrm{RbBr}^{\mathrm{T}}\right]^{+}[28,29,30]$. A band model for this photostimulation process is shown in Fig. 4 and discussed in more detail below. A typical PSL spectrum of BaFBr:Eu ${ }^{2+}$ is displayed in Fig. 2 as solid line. To separate the relevant F-center for the practically important excitation with a HeNe-laser at $632.8 \mathrm{~nm}$ the sample was first bleached at $625 \mathrm{~nm}$ and subsequently a second PSL spectrum was recorded (see dash-dotted line in Fig. 2). The difference of these two spectra is plotted as the dashed line and it was shown that this stimulation bands are typical for a bromine based $\mathrm{F}$-center $\left(\mathrm{F}\left(\mathrm{Br}^{-}\right)\right.$-center $)$in $\mathrm{BaFBr}[27$.

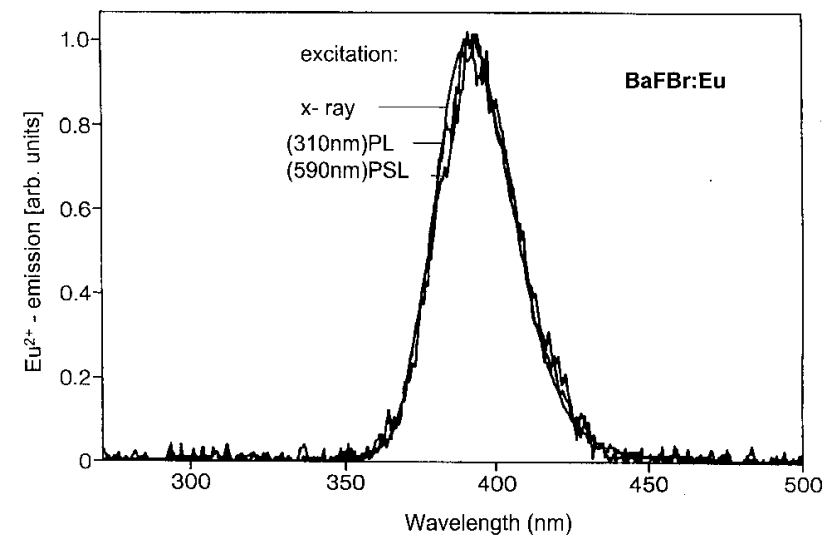

Figure 3. Comparison of $\mathrm{Eu}^{2+}$ emission spectra in BaFBr: $\mathrm{Eu}^{2+}$ for x-ray, PL- (310nm) and PSL-(590nm) excitation.

The electronic levels of the $\mathrm{F}\left(\mathrm{Br}^{-}\right)$-center are dis- played in Fig. 4. The center has two stimulation bands at $2.1 \mathrm{eV}$ and $2.5 \mathrm{eV}$, respectively. The excitation corresponds to an optical dipole allowed transition from a 1 s to a $2 \mathrm{p}$ state. From the physics of color centers it is known that the excited state, once occupied, undergoes a lattice relaxation within picoseconds due to the different charge distribution of the ground and the excited state. In Fig. 4 this is indicated by the dotted lines. The electronic energy of the relaxed excited state (RES) is found close to the conduction band so that the electron at low temperatures is still bound. For a release from the $\mathrm{F}$-center a thermal energy of $35 \mathrm{meV}$ [27] has to be available which is comparable to the alkali halides [30]. If this thermal energy is not supplied to the electron it will fall back into the F-center ground state via the non- relaxed ground state under the emission of infrared light.

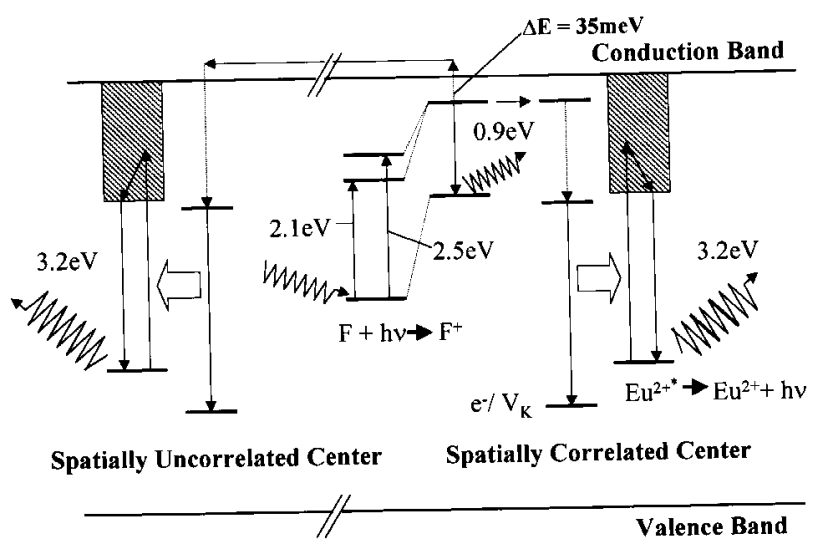

Figure 4. Bandmodel of the PSL process in BaFBr:Eu ${ }^{2+}$.

The hole storage center will now be addressed. In all investigated materials the photostimulated luminescence originates from the characteristic emission of the activator. In the case of $\mathrm{BaFBr}: \mathrm{Eu}^{2+}$ this corresponds to the $\mathrm{Eu}^{2+}$ emission [31] which is explained by a phonon broadened $4 f^{6} 5 \mathrm{~d} \rightarrow 4 \mathrm{f}^{7}$ transition. The typical spectrum for different excitation processes is displayed in Fig. 3 and resembles a recombination of the excited F-center electron with the $\mathrm{Eu}^{2+} /$ hole center of Fig. 4. By investigations of Hangleiter et al. [32] and Koshnick et al. [33] the $\mathrm{Eu}^{2+}$ ion captures the hole without the formation of $\mathrm{Eu}^{3+}$ as was believed initially [31]. This result is in agreement with other groups [34]. In this respect it is interesting to notice that the spectral dependence of the excitability of $\mathrm{Eu}^{2+}$ overlaps strongly with a detected exciton decay of an undoped $\mathrm{BaFBr}$ 
crystal [35]. This coincidence indicates the strong possibility of a resonant energy transfer between a bound exciton at the $\mathrm{Eu}^{2+}$ site and the $\mathrm{Eu}^{2+}$ ion. The resulting model for the emission is then as follows: $\mathrm{Eu}^{2+}$ captures a hole, in the present case a $V_{K}$ - or H-center, upon irradiation. After photostimulation and liberation of the F-center electron this electron is captured by the trapped hole forming a bound exciton. When deexciting, the exciton transfers its energy resonantly to the Europium ion. The Eu ${ }^{2+}$ ion will become excited and subsequently decays under the emission of its characteristic luminescence at $390 \mathrm{~nm}(3.2 \mathrm{eV})$. The scheme is also displayed in Fig. 4.

\section{III.2 Charge transport during PSL}

In this section the question concerning the mechanisms of charge transport is addressed. In this context the relaxed excited state of the $\mathrm{F}$-center plays a major role [27,36,37]. After photoexcitation the electron reaches the RES from where it can follow three different paths:

1) it escapes into the conduction band by accepting a thermal energy of $35 \mathrm{meV}$ from the lattice,

2 ) it tunnels to a neighboring hole center, or

3 ) it deexcites into the F-center ground state under the emission of an infrared photon $(0.9 \mathrm{eV})$.

Which one of the competing processes occurs depends on the spatial arrangement of the electron and hole storage centers, the temperature and the intrinsic lifetime of the electrons in the RES. The relative distance of the storage centers determines the probability of the tunneling and the temperature determines the escape of the electron into the conduction band.

Before we continue two expressions will be introduced: the spatially correlated and the spatially uncorrelated center. A spatially correlated center is defined as one where the transfer occurs by tunneling and the uncorrelated center is one where this is not the case. The definition is meaningful since for tunneling to occur, very small spatial distances between the involved electronic centers are required. In contrast to the uncorrelated centers, which do not contribute to the PSL, as soon as the thermal energy to escape into the conduction band is lacking within the lifetime of the RES the correlated centers continue to allow PSL to occur even to the lowest temperatures.

Under the assumption of these processes it was possible to classify the PSL centers [27]. By employing the temperature dependence of the escape of the RES electron to the conduction band all uncorrelated centers can be deactivated. In Fig. 5 the temperature dependence of the PSL intensity is displayed. In the corresponding experiment the sample was irradiated at room temperature and then cooled to the indicated temperatures. On the way down the information content was probed by a PSL experiment performed at very low stimulation light intensities. So no loss of PSL centers due to the experimental conditions were found. Therefore the observed step in Fig. 5 is solely due to the freezing-in of electrons in uncorrelated centers and not due to loss of the total number of centers. To further verify this result the sample was heated up again to temperatures as high as $120^{\circ} \mathrm{C}$ and no difference in the intensity amplitudes compared to the cooling-down phase was observed. The observation of an increasing infrared emission during this activation step in the cooling phase and a decreasing emission during the heating emphasizes the validity of this model. If an F-center without neighboring hole center is photo-excited and the RES electron cannot escape by thermal activation into the conduction band it can only relax into the $\mathrm{F}$-center ground state under emission of infrared light.

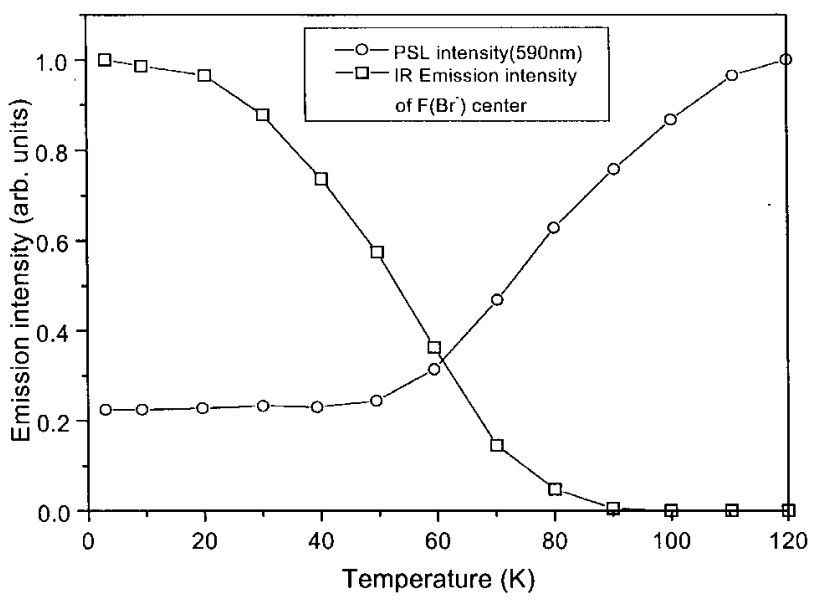

Figure 5. Temperature dependence of the PSL and IR emission intensities of a $\mathrm{BaFBr}: \mathrm{Eu}^{2+}$ single crystal after x-ray exposure at room temperature (x-ray dose: $5 \mathrm{R} ; V_{p p}: 70 \mathrm{kV}$; stimulation wavelength: $\lambda=590 \mathrm{~nm}$ ).

The ratio of the PSL intensity below the thermal activation step $(<40 \mathrm{~K})$ compared to the one above 
the activation step $(>120 \mathrm{~K})$ resembles the ratio of spatially uncorrelated to correlated centers. In the displayed example of a $\mathrm{BaFBr}: \mathrm{Eu}^{2+}$ crystal the ratio is $20 \%$. This ratio is found to strongly depend on the preparation. Examples between 10\% [32] and 85\% [37] were reported. The resulting transport processes are included in Fig. 4. The existence of correlated centers was verified by Koschnik et al. [38] using optically detected EPR-measurements which suggested a correlation to $\mathrm{O}^{-}$centers also observed in the material [39]. The existence of such correlated centers was already predicted by theoretical interpretations of experimentally observed PSL decay curves [36,40]. Similar investigations concerning the readout mechanism were performed on $\mathrm{RbBr}: \mathrm{Tl}^{+}$, and $\mathrm{RbI}: \mathrm{Tl}^{+}$, and similar processes were observed $[28,41]$.

\section{III.3 X-ray formation of PSL storage cen- ters}

The previously presented results concern the readout process and the nature of the electron and hole storage centers. Physically interesting, however, is the process by which these centers are generated. Two models are discussed. Both models start from different initial condition. In the first model it is assumed that the material contains a sufficient number of positively charged anion vacancies $\left(\mathrm{F}^{+}\right.$-centers) which become occupied by $\mathrm{x}$-ray generated electrons to form $\mathrm{F}$ - centers. The simultaneously generated holes, in this case the well known $V_{K}$-centers, are trapped by the $\mathrm{Eu}^{2+}$ centers to form recombination centers. The simplified reaction equations are

$$
\begin{gathered}
h \nu_{x-\text { ray }} \rightarrow e^{-}+V_{K}, \\
e^{-}+F^{+} \rightarrow F \\
V_{K}+E u^{2+} \rightarrow\left(V_{K} / E u^{2+}\right)-\text { complex } .
\end{gathered}
$$

The second model claims the absence of anion vacancies and assumes that the complete center is generated by the x-ray irradiation. This model was originally proposed by Itoh [42] for alkaline halides and was suggested by von Seggern to explain the processes in storage phosphors. According to Itoh the absorbed $\mathrm{x}$ ray energy forms excitons. Upon its decay the released energy displaces a $\mathrm{Br}$-atom from its regular lattice site leaving the electron behind. The Br-atom then combines with a neighboring $\mathrm{Br}^{-}$-ion to generate the well known $\mathrm{H}$-center, a $\mathrm{Br}_{2}^{-}$-center. The electron left behind is trapped in the bromine vacancy and establishes the $\mathrm{F}\left(\mathrm{Br}^{-}\right)$-center. The $\mathrm{H}$ - center then diffuses through the lattice and gets trapped at an $\mathrm{Eu}^{2+}{ }_{- \text {site generating the }}$ $\mathrm{Eu}^{2+} /$ hole complex acting as a recombination center.

The adequate reaction equations are

$$
\begin{aligned}
l l l h \nu_{x-\text { ray }} & \rightarrow\left(e^{-} / h\right) \text { exciton }, \\
\left(e^{-} / h\right) \text { - exciton } & \rightarrow F-H \text { pair } \\
F-H \text { pair } & \rightarrow F+\left(H / E u^{2+}\right)-\text { complex } .
\end{aligned}
$$

The model by von Seggern is supported by measurements at the HASYLAB (DESY) in Hamburg which showed a drastically increased production of PSLcenters when the material is excited within the exciton bands $[43,44,45]$ (see Fig. 6). Since the excitonic state is a major part of the Itoh model it has to be considered as part of the formation process. The model is further supported by the unsuccessful experiments to obtain a larger $\mathrm{F}^{+}$-center concentration by thermal quenching the material. Presently there is no clear proof for one or the other model. Due to the experimental evidence it seems, however, that the Itoh model is more appropriate.

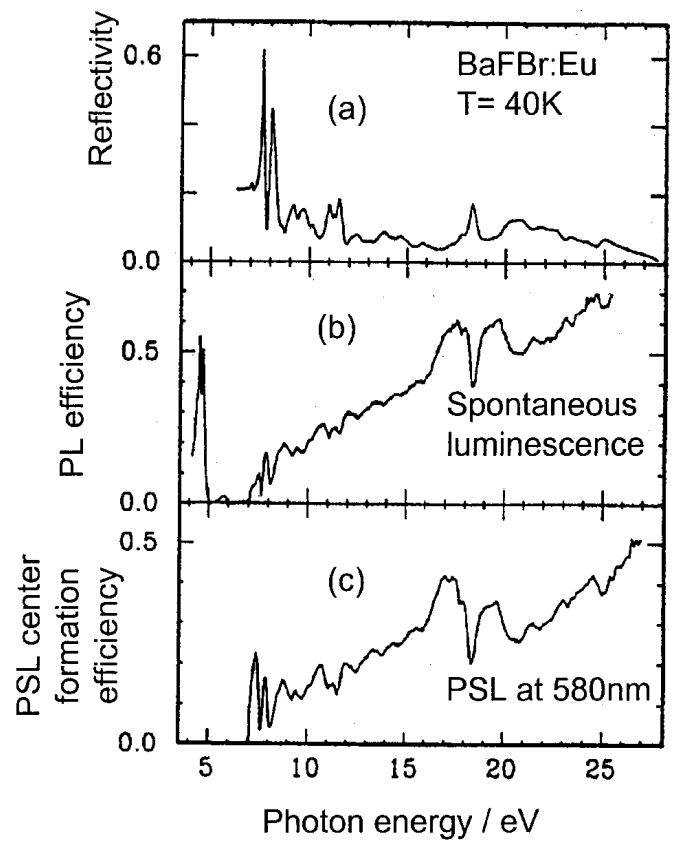

Figure 6. Comparison of reflectivity (a), spontaneous luminescence (b), and formation of photostimulable centers (c) in a $\mathrm{BaFBr}: \mathrm{Eu}^{2+}$-single crystal [23]. The relevant exciton bands are located at 7.4 and $8.0 \mathrm{eV}$. 


\section{Improved performance by new and modified storage phosphors}

Storage phosphor systems are commercially available but are mainly directed towards medical application. Since in this application the necessary x-ray dose is of major concern one has to compromise between the desired spatial resolution and the sensitivity of the image plate. In practice one accepts a reduced resolution in favor of a higher sensitivity. In the field of materials science these constraints are often relaxed.

Improvements can be separated in two types: (a) improvements of physical parameters such as stimulation wavelength and readout time and (b) improvement of image plate properties for PSL sensitivity and spatial resolution.

\section{IV.1 Photostimulation in the near- infrared}

As discussed in the previous chapters, $\mathrm{BaFBr}: \mathrm{Eu}^{2+}$ is stimulated at $590 \mathrm{~nm}$. For the HeNe-laser this wavelength is not optimal as can be seen in Fig. 2. It would therefore be desirable to shift the stimulation spectra more towards the red or to the near infrared since very powerful and small solid state lasers are available.

It is important to understand the relation between the stimulation wavelength and the properties of the electron trap. From the alkali halides such a relationship is known as the Mollwo relation [46]. It describes the dependence of the absorption energy and the physical size of the anion vacancy. This relation has its origin in the simple picture of an electron in a potential well in which the relation can be formulated as

$$
\Delta E=E_{2 p}-E_{1 s}=\frac{3 \pi^{2} \hbar^{2}}{8 m a^{2}},
$$

where $E_{1 s}$ and $E_{2 p}$ represent the energy in the ground state and the excited state, respectively. $m$ is the effective electron mass and $a$ the width of the potential well. One realizes that an enlargement of the potential well results in a reduction of the excitation energy and a shift of the exciting wavelength towards longer wavelength.

That this relationship qualitatively describes the red shift can be seen from storage phosphors made of alkali halides such as $\mathrm{RbBr}: \mathrm{Tl}^{+}$and $\mathrm{RbI}: \mathrm{Tl}^{+}$. In these materials the PSL stimulation spectrum follows the absorption spectra of the F-centers which is reported at 700nm [28,29,47] for RbBr:Tl ${ }^{+}$and at 780nm [41] for $\mathrm{RbI}: \mathrm{Tl}^{+}$. The latter material is very promising with respect to its physical properties, however, a thermal instability of information storage related to an extreme sensitivity to humidity makes its application difficult.

The influence of material modification on the photostimulation spectrum was shown in an article by Winnacker [48] in which he presented results on BaFBr:Ce ${ }^{3+}$ and $\mathrm{BaF}(\mathrm{Br}, \mathrm{I}): \mathrm{Ce}^{3+}$. An addition of $16 \mathrm{~mol}-\%$ of $\mathrm{BaI}_{2}$ to the regular preparation of $\mathrm{BaFBr}: \mathrm{Eu}^{2+}$ resulted in a large red shift of the stimulation spectrum (see Fig.7). The shift was explained by the implantation of the larger iodine ion in the bromine lattice which enlarges the average lattice spacing and therefore widens the $\mathrm{F}\left(\mathrm{Br}^{-}\right)$-centers. As indicated by the two laser lines, the modified storage phosphor can already be efficiently used in combination with a $670 \mathrm{~nm}$ GaAlAs solid state laser.

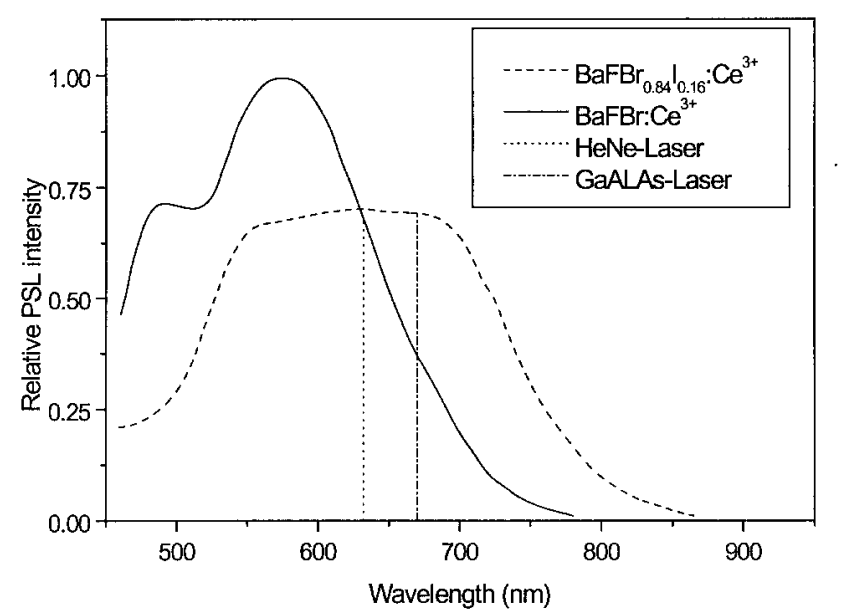

Figure 7. Photostimulation spectra of $\mathrm{BaFBr}: \mathrm{Ce}^{3+}$ and $\operatorname{BaF}\left(\mathrm{Br}_{0.84} \mathrm{I}_{0.16}\right): \mathrm{Ce}^{3+}[48]$.

A red shift in the photostimulation spectrum was also reported by Meyerink [49] and by Dietze et al. [50] who investigated nonstoichiometric crystals of $(\mathrm{Ba}, \mathrm{Sr}) \mathrm{F}_{1+x} \mathrm{Br}_{1-x}: \mathrm{Eu}^{2+}$ and $\left(\mathrm{Ba}_{1-x} \mathrm{Sr}_{x}\right) \mathrm{FBr}: \mathrm{Eu}^{2+}$. Upon the study by Meijerink, the properties of the storage phosphor changes drastically by varying the ratio of $\mathrm{F}^{-} / \mathrm{Br}^{-}$. According to the authors the nature of the storage center changes from fluorine based to bromine based F-centers. Further, the stimulation spectrum was observed to broaden in case of replacement of Barium 
by Strontium ions. Dietze et al. report that $F_{A}\left(\mathrm{Br}^{-}\right.$, $\mathrm{Sr}^{2+}$ ) centers are generated which exhibit an absorption band at lower energies and could explain the observed red shift for these materials.

\section{IV.2 Faster readout times}

For the practical use of the image plate system it is important to read out the imaged information in relatively short times. Today's systems are capable of cycle times of about $1 \mathrm{~min}$ [34] for the readout time and the erasure time. The readout time and the erasure time are influenced by two factors:

- the optical cross section of the photoexcited Fcenter and

- the lifetime of the photostimulated luminescence.

The first process is determined by the oscillator strength of the F-center and the second by the lifetime of the first excited state of the $\mathrm{Eu}^{2+}{ }_{-}$ion [37]. The first limitation is a practical limit determined by the available laser power. The second one is a fundamental limitation which limits the possible readout speed. If one scans too fast, the initially excited pixel will still emit light while the next pixel is excited leading to a degraded resolution.

\section{IV.2.1 Optical cross section of storage phosphors}

The practical readout time is limited by the optical cross section $\sigma_{0}$ and the laser intensity $I_{0}$. The readout time is related to these according

$$
\tau=\left(\sigma_{0} I_{0}\right)^{-1}
$$

where $\tau$ describes the time which is necessary to read out the information to the $\mathrm{e}^{-1}$-th value. It determines the average duration a laser has to stay on each pixel to obtain a acceptable image quality:

$$
N=N_{0} e^{-\Delta t / \tau} \Rightarrow \Delta t=\tau \ln \frac{N_{0}}{N},
$$

where $N_{0}$ is the total number of generated PSL-centers and $\mathrm{N}$ the number of erased centers due to photostimulation. For an optimal readout the optical cross section should be at the maxium of the excitation spectrum, and it should have a high absolute value. Since only HeNe lasers or solid state lasers are presently applied in commercial scanners, their low intensity causes the time constant in Eq.(5) to be a problem.

Optical cross sections of various storage phosphors are displayed in Table I and compared to theoretical data $[30,36]$ The experimentally determined values are by a factor of 3 to 5 larger than the theoretical data. One could speculate that this difference is caused by the local light intensity which strongly depends on the grain size and there with the scattering of the incident laser light. In this context the relatively small value of the cross section in BaFBr reported by DeLeeuw et al. [40] could also be related to a different grain size distribution.

The impact of the optical cross section and the laser intensity can be shown when calculating the time necessary to record a complete image. For a medical exposure of the thorax one uses film sizes of $40 \times 40 \mathrm{~cm}^{2}$ digitized in a pixel size of $100 \times 100, \mu \mathrm{m}^{2}$. For a normal exposure one has to read out about $20 \%$ of the generated PSL-centers to obtain a good image quality. Applying a $40 \mathrm{~mW}$ HeNe laser (as is done in commercial scanners) which impinges on a single pixel one obtains a laser intensity of $1.25 \times 10^{21}$ photons $/ \mathrm{cm}^{2}$. With these values Eqs.(4) and (5) result in a readout time for a single pixel of $\Delta t=2.54 \cdot 10^{-7} \mathrm{~s}$. For a complete image one would then need 4.1s. Using a solid state laser (e.g. a GaAlAs laser emitting at $780 \mathrm{~nm}$ ) and the matching x-ray storage phosphor like $\mathrm{RbBr}: \mathrm{Tl}^{+}$one could easily reduce the readout time by a factor of more than ten.

\section{IV.2.2 Lifetime reduction of storage phosphors}

In commercially available image plate systems it is the lifetime $\tau_{L}$ of the photostimulated luminescence which limits the performance. This luminescence lifetime was measured at room temperature to be $\tau_{L}=$ $750 \mathrm{~ns}[22,36,37]$ for $\mathrm{BaFBr}: \mathrm{Eu}^{2+}$. The laser spot should be focused for at least 3 time constants on each pixel to avoid smearing out of information. The readout time for the thorax image used above is 36s. Obviously, this value is much larger than the practical readout time and therefore resembles the bottleneck for a fast image processing time. 
Table I. Optical cross sections of various storage phosphors (original data)

\begin{tabular}{|c|c|c|c|c|}
\hline $\begin{array}{c}x \text {-ray voltage } \\
{[\mathrm{kV}]}\end{array}$ & $\begin{array}{c}\text { BaFBr } \\
{\left[\sigma_{0} / 10^{-16}\right] \mathrm{cm}^{2}}\end{array}$ & $\begin{array}{c}\operatorname{BaF}\left(\mathrm{Br}_{0,84} \mathbf{I}_{0.16}\right): \mathrm{Eu}^{2+} \\
{\left[\sigma_{0} / 10^{-16}\right] \mathrm{cm}^{2}}\end{array}$ & $\begin{array}{c}\text { RbBr:Tl }{ }^{+} \\
{\left[\sigma_{0} / 10^{-16}\right] \mathrm{cm}^{2}}\end{array}$ & $\begin{array}{c}\text { RbI:Ti }{ }^{+} \\
{\left[\sigma_{0} / 10^{-16}\right] \mathrm{cm}^{2}}\end{array}$ \\
\hline 40 & & 6.9 & 13.5 & 19.9 \\
\hline 78 & $2.0[36]$ & 6.9 & 12.8 & 19.3 \\
\hline theoretical & 0.86 & & 3.15 & 3.8 \\
\hline
\end{tabular}

In this chapter storage phosphors will be introduced which exhibit shorter PSL lifetimes. The lifetime limiting process must be investigated. Possible factors are the optical excitability of the electron trap, the thermal activation step, the trap-modulated charge transport in the conduction band, or the time delay induced by the recombination center itself. It was found that the rate limiting factor is introduced by the lifetime of the dopant in all investigated substances $[29,37,41]$. In case of $\mathrm{BaFBr}: \mathrm{Eu}^{2+}$ it was shown that the PSL lifetime and the photoluminescence lifetime do agree within the applied temperature range from room temperature down to LHe temperatures. Since in the photoluminescence experiment only the dopant is excited, this agreement indicates no other time limiting processes in the PSL.

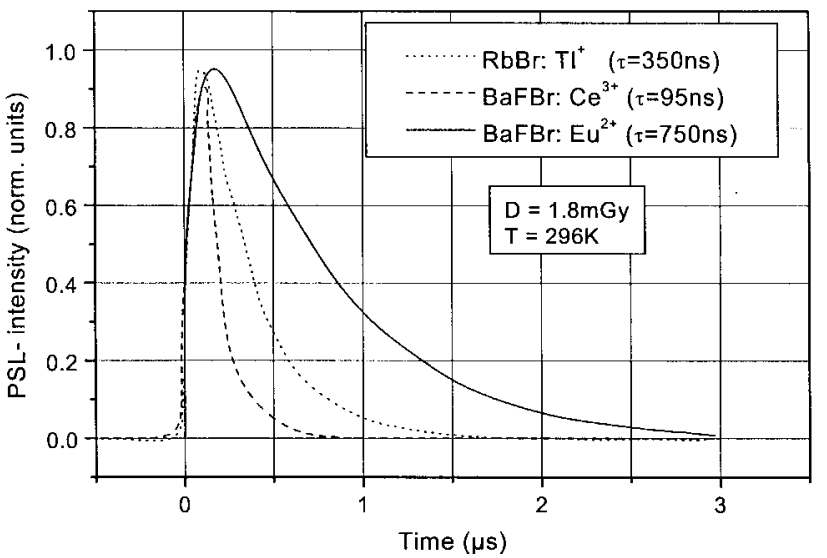

Figure 8. Comparison of the experimental PSL lifetime of BaFBr: $\mathrm{Eu}^{2+}$, BaFBr:Ce ${ }^{3+}$ and $\mathrm{RbBr}^{2} \mathrm{Tl}^{+}$after pulse excitation (10ns) at $590 \mathrm{~nm}[48]$.

In order to decrease the PSL lifetime a dopant with a shorter inherent lifetime has to be used. The only available candidate is the $\mathrm{Ce}^{3+}$-ion which is known to exhibit a lifetime shorter than 100ns. A comparison of the PSL lifetimes of both the $\mathrm{Eu}^{2+}$ and the $\mathrm{Ce}^{3+}$ doped BaFBr is displayed in Fig. 8 [48]. The resulting lifetime was measured to be $95 \mathrm{~ns}$ [48]. For the image readout time this resembles an improvement by a factor of 8 reducing the above discussed 36 s for a complete image readout to $4.5 \mathrm{~s}$. This value matches the practical limits for the present system. To fully utilize the existing laser power of solid state lasers at $670 \mathrm{~nm}$, the PSL lifetime has to be decreased by another factor of at least ten (see Eqs. 4 and 5). In this context it is interesting to mention that different dopants in RbI result in different lifetimes. A collection of all measured values in RbI is shown in Table II. The lifetimes extend from 350ns for a Thallium doped material to $3 \mu$ s for an Indium doped one [41].

\section{PSL-efficiency and informa- tion stability}

Since the PSL efficiency is one of the major performance factors of a detector system the present understanding of parameters determining this efficiency will be discussed. The PSL efficiency is influenced by:

- absorption of the primary radiation,

- conversion to PSL centers,

- efficiency of readout,

- structure of the image plate (scattering of stimulating light), and

- collection of PSL photons.

The conversion efficiency of $\mathrm{BaFBr}: \mathrm{Eu}^{2+}$ will be discussed and an estimate of further improvements of the $\mathrm{BaFBr}: \mathrm{Eu}^{2+}$ image plates will be presented. In order to be able to estimate the existing efficiency, the detection of x-ray generated non-photostimulated centers will be discussed first. 
Table II: Emission wavelength and PSL lifetimes of differently doped RbI samples at 300K [41]

\begin{tabular}{|c|c|c|}
\hline Dopant & Emission wavelength [nm] & PSL lifetime [ $\boldsymbol{\mu s}]$ \\
\hline $\mathrm{Tl}^{+}$ & 430 & 0.41 \\
\hline $\mathrm{In}^{+}$ & 540 & 3.0 \\
\hline $\mathrm{Pb}^{2+}$ & 425 & 1.2 \\
\hline $\mathrm{Eu}^{2+}$ & 425 & 1.3 \\
\hline
\end{tabular}

V.1 Non photostimulated centers in phors. BaFBr:Eu ${ }^{2+}$

In alkaline halides F-centers and higher agglomerates are generated by ionizing radiation [25,30]. In storage phosphors a variety of centers could be detected by their inherent infrared emission at low temperatures. Such an emission $(0.9 \mathrm{eV})$ is included in the term scheme of a $\mathrm{F}\left(\mathrm{Br}^{-}\right)$-center in Fig. 4. After determination of the different infrared emission bands, the nonphotostimulated centers can be identified by their characteristic excitation spectrum. Since excitation spectra of F-centers and of their aggregates are known in the case of the alkaline-halides, this knowledge can easily be transfered to the alkaline-earth halide storage phos-

A list of identified centers is compiled in Table III. Details concerning these measurements are found in [27]. The M-center in Table III is an agglomerate of two F-centers whereas the $\mathrm{F}_{A^{-}}$center is an agglomerate of a F-center and an activator ion, in our case a F-center close to an $\mathrm{Eu}^{2+}$-ion. The relative concentrations of these centers were determined by integrating the excitation spectra [27]. One should, however, notice that the values are based on the assumption that the different centers exhibit similar oscillator strength which is true for centers in alkaline- halides [30]. The obtained concentrations of these centers are listed in Table IV.

Table III Correlation of center types, infrared emission bands and excitation bands (Data taken at 4K) [27]

\begin{tabular}{|c|c|c|}
\hline IR emission peak $\lambda[\mu \mathbf{m}]$ & Excitation peak $\lambda[\mathbf{n m}]$ & Correlation \\
\hline 1.38 & 495,590 & $\mathrm{~F}(\mathrm{Br})$ \\
\hline 1.10 & 370,512 & $\mathrm{~F}\left(\mathrm{~F}^{-}\right)$ \\
\hline 1.10 & 712 & $\mathrm{M}\left(\mathrm{F}^{-}\right)$ \\
\hline 1.6 & 445,520 & $\mathrm{~F}_{\mathrm{A}}\left(\mathrm{Eu}^{2+}, \mathrm{F}^{-}\right)$ \\
\hline 1.6 & 680 & $\mathrm{M}_{\mathrm{A}}\left(\mathrm{Eu}^{2+}, \mathrm{F}^{-}\right)$ \\
\hline
\end{tabular}

Table IV: Relative concentration and creation efficiency of radiation induced defects and the of spontaneous luminescence photons in a BaFBr: $\mathbf{E u}^{2+}$-crystal $[27,51]$

\begin{tabular}{|c|c|c|c|}
\hline Center types & $\begin{array}{c}\text { Relative concentration } \\
{[\%]}\end{array}$ & $\begin{array}{c}\text { Generation rate } \\
\text { [number } / \mathrm{keV}]\end{array}$ & $\begin{array}{c}\text { photostimulated } \\
\text { yes/no }\end{array}$ \\
\hline $\mathrm{F}_{\text {uncorr }}\left(\mathrm{Br}^{-}\right)$ & 12.4 & 6.16 & yes \\
\hline $\mathrm{F}_{\text {corr }}\left(\mathrm{Br}^{-}\right)$ & 3.7 & 1.82 & yes \\
\hline $\mathrm{F}^{\left(\mathrm{F}^{-}\right)}$ & 13.8 & 6.86 & no \\
\hline $\mathrm{F}_{\mathrm{A}}\left(\mathrm{Eu}^{2+}, \mathrm{F}^{-}\right)$ & 15.6 & 7.75 & no \\
\hline $\mathrm{M}\left(\mathrm{F}^{-}\right)$ & 39.0 & 10.36 & no \\
\hline $\mathrm{M}_{\mathrm{A}}\left(\mathrm{Eu}^{2+}, \mathrm{F}^{-}\right)$ & 4.8 & 2.35 & no \\
\hline luminescence photon & 10.7 & 5.21 & no \\
\hline
\end{tabular}




\section{V.2 Formation energy of centers in BaFBr: $\mathrm{Eu}^{2+}$}

The above results were used to estimate the energy efficiency of a BaFBr:Eu ${ }^{2+}$-crystal [51]. The energy necessary to generate a PSL center was determined following the flux diagram shown in Fig. 9. Taking into account the energy spectrum of the $x$-ray tube and the absorption and energy conversion coefficients, the absorbed energy was calculated. This energy was compared to the spontaneously emitted photons during $\mathrm{x}$ ray irradiation which leads to a formation energy of 129 $\mathrm{eV}$ / spontaneously emitted photon. Then a comparison was performed between the number of PSL photons and spontaneously emitted photons which accounts to a ratio of 1.53 . Taking this value and the concentration of non photostimulable centers (see Table IV) into account one obtains a value of $21 \mathrm{eV} / \mathrm{x}$-ray generated center. It is assumed that the formation energy of each center is the same. In addition, it is assumed that the oscillator strength of the different centers is the same. This is a good assumption since all centers are generated by the Itoh process or by an ionization process. In both cases every center creation process starts with an elementary process of generating an exciton or equivalently an highly excited electron. The assumption of the same value for the oscillator strength for all centers is also acceptable [30].

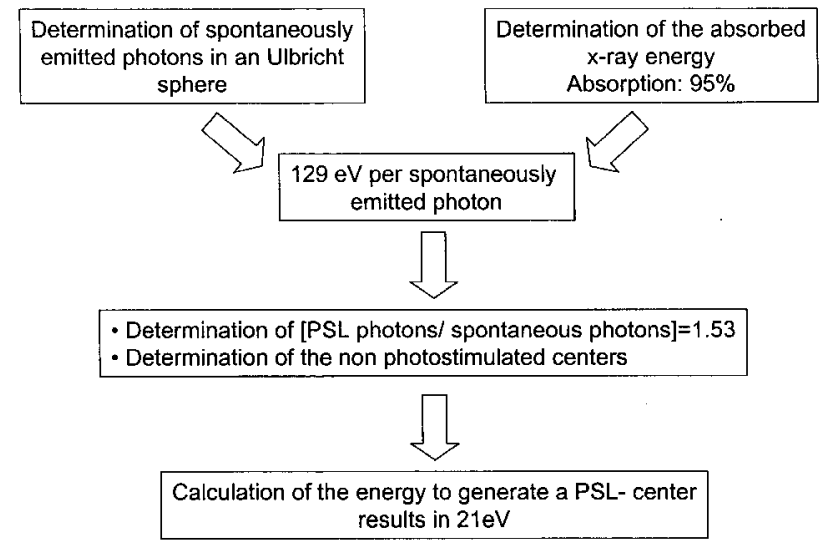

Figure 9. Flux diagram of the energy balance of the generation of a PSL center in $\mathrm{BaFBr}: \mathrm{Eu}^{2+}$.

The formation energy of $21 \mathrm{eV}$ per center is very low with respect to the gap energy of $8.3 \mathrm{eV}$ in $\mathrm{BaFBr}: \mathrm{Eu}^{2+}$. The value for generating an electron hole pair in a semiconductor or insulator amounts to about 3 times the bandgap. Under the above assumptions the totally absorbed energy is efficiently used to generate defect cen- ters. For a further improvement of BaFBr:Eu ${ }^{2+}$ the only way is to reduce the number of non photostimulated centers or the number of spontaneously emitted photons. The number of $16 \%$ of photostimulated centers allows further improvement.

\section{Performance parameters}

Image quality plays a major role in the performance of image plate systems. Image quality is determined by the following factors:

- spatial resolution,

- image noise or homogeneity, and

- PSL sensitivity.

The first two factors are determined by the structure of the layer whereas the latter is determined by both the structure of the plate and the material properties. The principle of light scattering in image plates is displayed in Fig. 10. This figure illuminates the structural reasons for the spatial resolution and the image noise of the plates. The image plate consists of a storage phosphor powder imbedded in an organic binder which is deposited on an organic substrate. Due to the different refractive indices of both components in the active layer, the stimulating light is scattered within the plate until it is absorbed by a F-center or leaves the sample. Therefore the spatial resolution is determined by the excitation volume of the stimulating light. The resulting PSL is detected globally by means of a modified Ulbricht sphere and therefore does not contribute to the spatial resolution, but to the PSL sensitivity of the readout system. The homogeneity of the image plate is determined by the variation of the total amount of the storage phosphor per pixel and, in addition, to the local sensitivity of the phosphor grains in each pixel. This means that the fluctuations in the grain size and local storage phosphor density generate noise when scanning the image plate with the readout laser. 


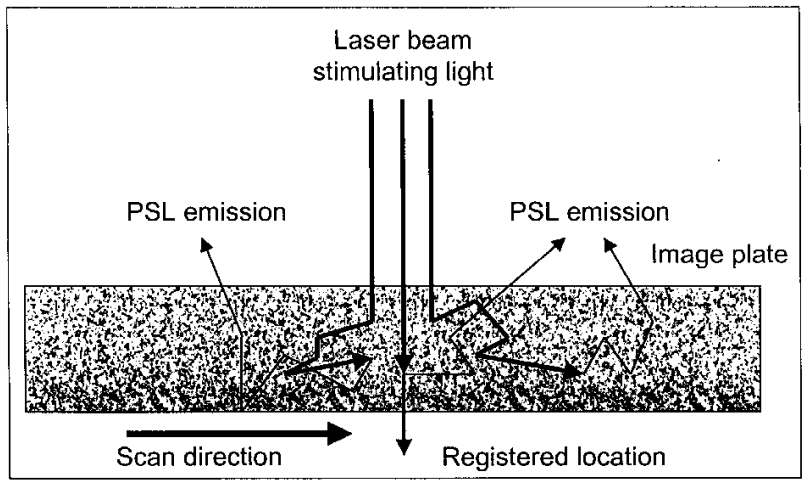

Figure 10. Schematics of propagation of photostimulation and emitted PSL light in an image plate.

These image parameters are investigated in a number of publications [52-58] dealing with commercially available image plates. A study on ceramic storage phosphors [59], found performance similar to the classical plates. Recently, theoretical models estimated the influence of light propagation on the image plate performance [60-62]. One model was based on light propagation by a diffusion model $[60,61]$, and the other modeled the light propagation by a Monte Carlo technique [62]. Both experiments were able to qualitatively describe the image parameters.

\section{Applications}

In Fig. 11 an overview of today's and future fields of application are shown $[63,64]$. Commercial systems are available for medical diagnostics $[22,65,66]$, for general crystallography [67-70], for protein crystallography $[71,72]$ and for auto-radiography [73]. New applications have been suggested in the field of data storage [74], electron microscopy [75], detection of soft x-rays [76], and in the field of dosimetry [77].

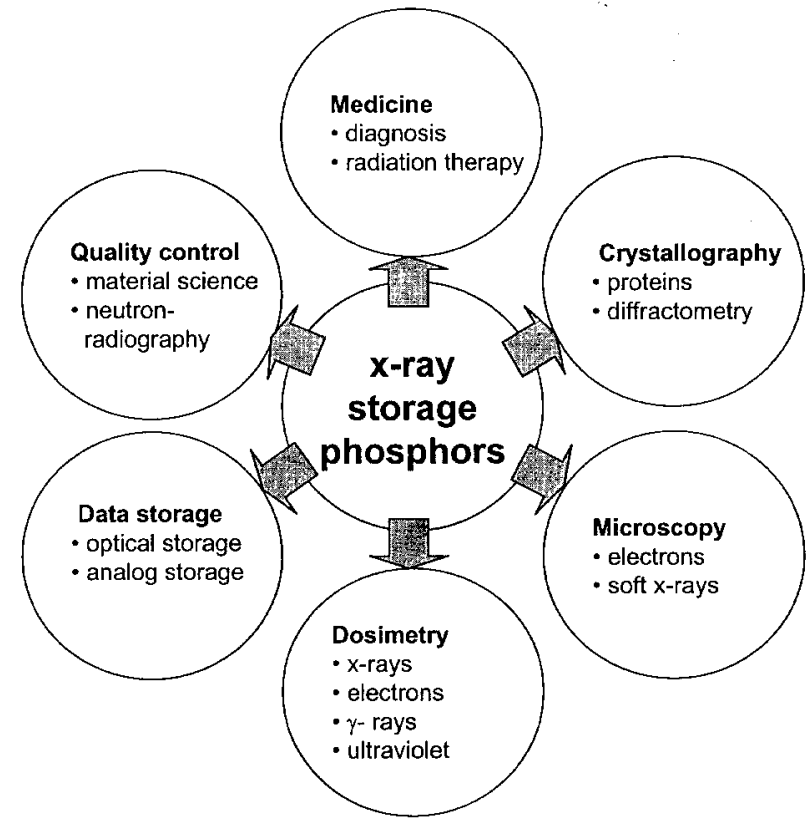

Figure 11. Present and future applications of x-ray storage phosphors.

Close to commercial application is the utilization of image plates as thermal neutron detectors. This method is interesting since it offers complementary information compared to the x-ray application. Since commercial image plates exhibit a too small absorption for neutrons, they were initially combined with a gadolinium metal film which strongly absorbs thermal neutrons [78]. The gadolinium is a neutron converter with a very large cross section of 49700 barns/atom. By an $(n, \gamma)$-reaction, energetic electrons are liberated with energies between 29 and $130 \mathrm{keV}$ which then expose the image plate. This, however, leads to a drastically reduced spatial resolution [78,79].

The problem was eliminated by introducing a uniform mixture of $\mathrm{Gd}_{2} \mathrm{O}_{3}$ powder of $2 \mu \mathrm{m}$ grain size into the image plate [78,80-82]. The oxide, instead of the metal, allows the resulting PSL to escape from the image plate. The results are very promising and are collected in Table V. The Fuji Film Company, Japan, announced a coomercial film on the International Workshop on Neutron Detection at the Brookhaven National Laboratories in 1995. Rausch et al. have recently built up a tomographic neutron detector based on neutron image plates [83]. 
Table V: Comparison of the $\mathrm{Gd}_{2} \mathrm{O}_{3}$-storage phosphor neutron detector with conventional thermal neutron detectors $[80]$

\begin{tabular}{|c|c|c|c|}
\hline Neutron detector & $\begin{array}{c}\text { Detective quantum } \\
\text { efficiency [\%] }\end{array}$ & $\begin{array}{c}\text { Spatial } \\
\text { resolution } \\
\text { FWDH }[\mu \mathrm{m}]\end{array}$ & $\begin{array}{c}\text { Dynamical range in } \\
\text { neutron dose in } \\
\text { [orders of magnitude] }\end{array}$ \\
\hline $\mathrm{Gd}_{2} \mathrm{O}_{3}$-storage phosphor & 18.2 & 80 & $>4$ \\
\hline $\mathrm{Gd}$ foil / image plate & 6.3 & 240 & $>4$ \\
\hline $\mathrm{Gd}$ foil/ photographic film & 1.7 & 20 & $2-3$ \\
\hline
\end{tabular}

\section{Outlook}

$\mathrm{X}$-ray storage phosphors have already reached a high level of performance with commercial applications. One looks forward to future improvements and new applications. The large number of non-photostimulated centers, it seems, still can be improved with respect to sensitivity and spatial resolution.

Today's films are tuned towards medical application which implies a compromise between spatial resolution and PSL sensitivity. For applications in materials science and quality control the requirements are different. In quality control the demand for a higher spatial resolution is essential which means that the grain size of the phosphor has to be reduced or new layer techniques have to be developed. For applications in materials science, such as diffractometry the need for more quantitative data is increasing.

Since today's image plates have a relatively large temporal loss of information after exposure one has to search for mechanisms to stabilize the recorded information. This is especially important for applications which require a long term exposure. Scientifically, the question of the nature of the hole traps is still under debate, as is the question of the generation mechanism of the PSL centers. New ideas are necessary to identify the possible radiation products during center formation such as the $\mathrm{V}_{K}$-centers and the $\mathrm{H}$-centers.

\section{References}

1.. B. Gross, Zeitschrift fuer Physik 78, 271 (1932).

2.. B. Gross, Zeitschrift fuer Physik 83, 214 (1933).
3. B. Gross, An. Acad. Bras. Ci Symposium sobre raios cósmicos, 171 (1941).

4. B. Gross, An. Acad. Bras. Ci 20, 4 (1948).

5. B. Gross, Metrology of radionuclides IAEA, 413 (1960).

6. B. Gross, P.V. Murphy, Nucleonics 19, 86 (1961).

7. B. Gross, J. Radiation Research 14, 117 (1961).

8. B. Gross, P.V. Murphy, Selected Topics in Radiation Dosimetry IAEA, 549 (1961).

9. B. Gross, An. Acad. Bras. Ci, Comunicação 40, 3 (1968).

10. B. Gross, US-Patent 3122640, (25.Feb.1964).

11. B. Gross, Nucleonic 6, 20 (1964).

12. R. Gregorio Filho, B. Gross, J. Appl. Phys. 66, 5478 (1989).

13. B. Gross, C.A.F. Pintao, R Hessel, to be published in this issue.

14. B. Gross, J E. West, H. von Seggern, D.A. Berkley, J. Appl. Phys. 51, 4875 (1980).

15. B. Gross, H. von Seggern, D.A. Berkley, Conf. on Electrical Insulation and Dielectric Phenomenon, 206, (1982).

16. B. Gross, H. von Seggern, D.A. Berkley, Phys. Stat. Sol. 79, 607 (1983).

17. B. Gross, H. von Seggern, J.E. West, D.A. Berkley, Conf. on Electrical Insulation and Dielectric Phenomenon, IEEE, 227, (1984).

18. B. Gross, H. von Seggern, J.E. West, J Appl. Phys. 56, 2333 (1984).

20. D. Zweig, J. Zweig, Imaging Techn. 10, 43 (1984).

21. P.C. Bunch, K E. Huff, R. van Metter, SPIE Vol. 626, Medicine XIV/PACS IV, 64 (1986).

22. M. Sonoda, M. Takano, J. Miyahara, H. Kato, Radiology 148, 833 (1983).

23. A. Meijerink, G. Blasse, J. Phys. D: Appl. Phys. 24, 626 (1991).

24. A. Meijerink, W.J. Shipper, G. Blasse, H. Phys. D: Appl. Phys. 24, 997 (1991).

25. J.J. Markham, F-Centers in Alkali Halides, (Academic Press, New York 1966). 
26. M. Yuste, L. Taurel, M. Rahmani, D. Lemyne, J. Phys. Chem. Solids 37, 961 (1976).

27. M. Thoms, H. von Seggern, A. Winnacker, Phys Rev. B44, 997 (1991).

28. K. Amitani, A.Kano, H. Tsuchino, F. Shimida, SPIE Conf. and Exhibition: Electric Imaging, 26th Fall Symposium 1986, Printing of Paper Summaries, 180.

29. H. von Seggem, A. Meijerink, T. Voigt, A. Winnacker, J. Appl. Phys. 66, 4418 (1989).

30. B. Fowler, in Physics of Cokrur Centers, edited by W.B. Fowler (Academic Press, New York 1968).

31. K. Takahashi, K. Kohda, J. Miyahara, J. Luminescence 31\&32, 266 (1984).

32. T. Hangleiter, F.K. Koshnick, J.-M. Spaeth, RH.D. Nuttal, R.S. Eachus, J. Phys. Condens. Matter 2, 6837 (1990).

33. F.K. Koshnick, J.-M. Spaeth, R.S. Eachus, W.G. Mc Dugle, R.H.D. Nuttal, Phys. Rev. Lett. 67, 3571 (1991).

34. M. Thoms, H. Burtzlaff, A. Kinne, H. von Seggem, R. Spengler, A. Winnacker, Materialis Science Forum 228-231, 107 (1996).

35. H.H. Rueter, H. von Seggem, R. Reininger, V. Saile, to be published in J. Appl. Phys.

36. H. von Seggem, T. Voigt, W. Knuepfer, G. Lange, J. Appl. Phys. 64, 1405 (1988).

37. H. von Seggem, Cryst. Latt. Def. and Amorph. Mat. 18, 99 (1989).

38. F.K. Koshnick, J.-M. Spaeth, R.S. Eachus, J. Phys.: Condens. Matter 4, 8019 (1992).

39. F.K. Koshnick, J.-M. Spaeth, R.S. Eachus, J. Phys.: Condens. Matter 4, 3015 (1992).

40. D.M. DeLeeuw, T. Kovats, S.P. Herko, J. Electrochem. Soc. 134, 491 (1987).

41. M. Thoms, H. von Seggem, A. Winnacker, J. Appl. Phys. 76, 1800 (1994).

42. N. Itoh, Adv. Phys. 31, 491 (1982).

43. H.H. Rueter, H. von Seggem, R. Reininger, V. Saile, Phys. Rev. Lett. 65, 2438 (1990).

44. V.V. Mikhailin, Nuc. Instr. Meth. 261, 107 (1987).

45. E. Nicklaus, Phys. Stat. Sol. (A). 53, 217 (1979).

46. E. Mollwo, Goett. Nachrichten 1931, 97 (1931).

47. K. Amitani, A. Kano, H. Tsuchino, F. Shimada, SPE's Conf. And Exhib.: Electric imaging, 26th Fall Symposium, 1986, Printing of Paper Summaries 180 .

48. A. Winnacker, Physica Medica, Vol. IX, N.2-3, 95, April-September 1993.

49. A. Meijerink, Materials Chemistry and Physics 44, 170 (1996).

50. C. Dietze, Th. Hangleiter, P. Willems, P.J.R.
Leblans, L. Struye, J.-M. Spaeth, J. Appl. Phys. 80, 1074 (1996).

51. M. Thoms, H. von Seggem, J. Appl. Phys. 75, 4658 (1994).

52. A.R. Lubinsky, J.F. Owen, D.M. Kom, SPIE Vol. 626, Medicine XIV/PACSIV, 120 (1986).

53. A.R. Lubinsky, B.R. Whiting, J.F. Owen, SPIE Vol. 767, Medical imaging, 167 (1987).

54. K. Klingenbeck, B. Conrad, Siemens Forschungsund Entwicklungsbericht 16, 192 (1987).

55. W. Hillen, U. Schiebel, T. Zaengel, Med. Phys. 14, 744 (1987).

56. M. Tecotzky, C. Andrew-Eurglunes, SPE Vol. 914, Medical Imaging II, 143 (1988).

57. R.H. Templer, N.A. Warrender, J.M. Seddon, J.M. Davis, Nuc. Instr. Meth. A310, 232 (1991).

58. R.H. Templer, Nuc. Instr. Meth. A300, 357 (1991).

59. BMBF-Final Report "Development of ceramic $\mathrm{x}^{-}$ ray storage phosphors" Proj. No. $03 \mathrm{M} 2707$ (1993).

60. M. Thoms, Applied Optics 35, 3702 (1996).

61. M. Thoms, H. von Seggern, J. Appl. Phys. 81, 5887 (1997).

62. R. Fassbender, A. Winnacker, to be published.

63. H. von Seggern, Physikalische Blatter 48, 719 (1992).

64. H. von Seggern, Nuc.Instr.Meth.: Phys. Research. A 322, 467 (1992).

65. "Konica Direct Digitizer KD-1000", a product of Konica working with RbBr:Tl+.

66. G. Witte, B. Schwemmer, E. Buecheler, Deutsches Aerzteblatt 86, A-2539 (1989).

67. Y. Amemiya, S. Kishimoto, T. Matsushita, Y. Satow, M. Ando, Rev. Sci. Instr. 60, 1552 (1989).

68. B.R. Whiting, J.F. Owen, R.H. Rubin, Nuc. Instr. Meth. A266, 628 (1988).

69. H. von Seggern, Materials Science Forum 79-82, 383 (1991).

70. Announcement of a Guinier Diffractometer by $\mathrm{Hu}^{-}$ ber Diffraktionstechnik GmbH, Germany.

71. J. Hendrix, Rev. Sci. Instr. 63, 641 (1992).

72. J. Miyahara, K. Takahaschi, Y. Amemiya, N. Kamiya, Y. Satow, Nuc. Instr. Meth. A246, 572, (1986).

73. Y. Amemiya, J. Miyahara, Nature 336, 89 (1988).

74. J. Lindmayer, Solid State Technol. 137, (Aug. 1988).

75. N. Mori, T. Oikawa, Y. Harada, J. Miyahara, J. Electron. Microsc. 39, 433 (1990).

76. P. Witt, Pure Appl. Opt. 2, 61 (1993).

77. A.R. Lakshmanan, K.G. Rajan, Protection 
Dosimetry 55, 247 (1994).

78. Ch. Rausch, T. Buecherl, R. Gaehler, H. von Seggern, A. Winnacker, SPE Vol. 1737 Neutrons, XRays and Gamma Rays, 255 (1992).

79. C. Wilkinson, SPE Vol. 1737 Neutrons, X-Rays and Gamma Rays, 324 (1992).

80. T. Buecherl, Ch. Rausch, H. von Seggern, Nuc. Instr. Methods A333, 502 (1993).

81. H. von Seggern, K. Schwarzmichel, T. Buecherl,
Ch. Rausch, Materials Science Forum 166-169, 267 (1994).

82. M. Thoms, M.S. Lehmann, C. Wilkinson, Nucl. Instr. Meth. Phys. and Research A384, 457 (1997).

83. Ch. Rausch, T. Bcherl, J. Hofmann, B. Schillinger, H. von Seggern, Proc. 5th World Conf. Neutron Radiography, Berlin, 113-117 (1996). 